\title{
TB financing in East Europe promotes unnecessary hospital admissions: the case of Armenia
}

\author{
Laura Gillini ${ }^{1}$, Karapet Davtyan ${ }^{2}$, Hayk Davtyan ${ }^{3}$, Armen Hayrapetyan ${ }^{4}$, Sergey Khachatryan ${ }^{5}$, Rosella \\ Centis $^{6}$, Lia D’Ambrosio ${ }^{6}$, Masoud Dara ${ }^{7}$, Giovanni Battista Migliori ${ }^{6}$, Giovanni Sotgiu ${ }^{8}$ \\ ${ }^{1}$ Health system strengthening Armenia HSSTAR Abt/USAID grantee Armenia \\ ${ }^{2}$ National Tuberculosis Control Office, Armenia \\ ${ }^{3}$ Monitoring and Evaluation Department of National TB Control office, Armenia \\ ${ }^{4}$ National TB Program, Armenia \\ ${ }^{5}$ Ministry of Health, Armenia \\ ${ }^{6}$ World Health Organization Collaborating Centre for Tuberculosis and Lung Diseases, Fondazione S. Maugeri, \\ Care and Research Institute, Tradate, Italy \\ ${ }^{7}$ World Health Organization, Regional Office for Europe, Copenhagen, Denmark \\ ${ }^{8}$ Epidemiology and Medical Statistics Unit, Department of Biomedical Sciences, University of Sassari - Research, \\ Medical Education and Professional Development Unit, AOU Sassari, Italy
}

Key words: tuberculosis; MDR-TB; XDR-TB; Armenia; financing

J Infect Dev Ctries 2013; 7(3):289-292.

(Received 11 February 2013 - Accepted 25 February 2013)

Copyright (C) 2013 Gillini et al. This is an open-access article distributed under the Creative Commons Attribution License, which permits unrestricted use, distribution, and reproduction in any medium, provided the original work is properly cited.

\section{Background}

Based on cost-effectiveness considerations and on the risk of nosocomial transmission of Mycobacterium tuberculosis strains, the World Health Organization (WHO) and other international institutions recommend to minimize unnecessary hospitalization of tuberculosis (TB) cases [1,2,3,4].

As in other Former Soviet Union (FSU) countries, the organisations of TB services in Armenia are heavily based on vertical and specialized services.

In Armenia, a country of 3.2 million inhabitants, $\mathrm{TB}$ is one of the major public health problems with overall notification and estimated mortality rates of 41 and 8.8 cases per 100,000 population in 2011, respectively $[5,6]$. The prevalence of multidrugresistant (MDR-TB) was $9.4 \%$ and $43.2 \%$ among new and previously treated cases, respectively, with $11.9 \%$ of them estimated to be extensively drug-resistant (XDR-TB) [6,7].

Specialized TB services include 9 in-patient TB departments, 73 out-patient TB units (called TB cabinets) based in general health-care facilities, and 31 laboratories providing sputum smear microscopy, while culture and drug susceptibility testing is performed in the National TB Reference Laboratory.
Following WHO recommendations, the Armenian National TB Programme (NTP) expanded the basic package of services provided in out-patient settings, allowing free access/free of charge TB diagnosis and treatment to the population, to achieve the global TB targets in the WHO European Region under the National Strategic Plan 2007-2015 and Consolidated Action Plan to Prevent and Combat M/XDR-TB in the WHO European Region 2011-2015 [8].

Despite programme success in reducing the default rate of new sputum smear-positive pulmonary $\mathrm{TB}$ patients from $14 \%$ to $7 \%$ in five years, the treatment success rate $(72 \%$ in 2011$)$ of the diagnosed cases is far below WHO targets [6]. The main shortcomings identified by the WHO Programme Review conducted in 2011 [7] include an alarming prevalence of $\mathrm{M} / \mathrm{XDR}-\mathrm{TB}$, suggesting that directly observed therapy (DOT) is not systematically applied. An analysis of risk factors for MDR-TB in Armenia proved that after completion of the hospital phase of treatment, a relevant proportion of patients $(6.1 \% ; 8 / 133)$ interrupts treatment during the ambulatory phase, with less than $30 \%$ of them undergoing DOT [9].

In addition, TB case detection for new sputum smear-positive pulmonary TB patients remains as low 
as $27 \%$. To improve programme performance and expand patient-centered approaches, it is important to strengthen outpatient services to improve TB detection and treatment adherence in Armenia. For the abovementioned reasons, the WHO Programme Review has begun to promote ambulatory diagnosis and treatment for TB patients to reduce unnecessary hospitalizations and excessive costs [7].

The costs of the TB services, such as staff salaries, in-patient and ambulatory care costs, are largely covered by the government, with most of the health expenditure represented by recurrent costs $(96.7 \%$ in 2011) versus capital costs (3.3\%) [9]. The Global Fund supports diagnosis and treatment to manage M/XDRTB cases.

The current funding scheme for hospital-based care for TB relies on bed occupancy/days. The cost of one bed per day in 2012 was 8,900 Armenian Drams (AMD) $(\approx 20 €)$, while the cost of one TB cabinet visit is about 5.6 times cheaper (about 1,600 AMD $\approx 3 €$ ) [10].

The refund related to the out-patient activities is fixed, based on the catchment population of the facility, so that staff is not motivated to increase the daily number of medical visits. In other words, physicians, receiving a fixed salary, are not encouraged to manage TB cases in an out-patient setting, and prefer to refer them for admission. As a consequence, $80 \%$ of the TB case-load is absorbed by the in-patient system against $20 \%$ managed by the ambulatory system. Unfortunately, this mechanism creates an unfair competition for resources between the "old" hospital-centred and the "new" patientcentred model. The Ministry of Health is considering the options to revise the funding mechanism for TB services; however, no changes have been instituted as of today.

The aims of the present study are to 1) evaluate the effect of maintaining the present funding scheme based on in- versus out-patient management, and 2) estimate the savings that might be achieved limiting the unnecessary hospitalizations of non-infectious (sputum smear-negative and extrapulmonary) TB cases.

\section{Methodology}

A retrospective analysis of monitoring and evaluating data from the newly implemented NTP electronic database was performed using national data for 2011 and 2012 (first six months).

In addition to quarterly routine recording and reporting notifications, a standard questionnaire used by TB supervisors during their monitoring visits to document referral to hospital-based care and in- versus out-patient management was analyzed. Indicators of TB care management (2011 versus 2012) were calculated and compared using Epi Info 7 statistical software. Chi square test was used to test qualitative variables.

\section{Results}

A consistent number of TB suspects is still referred to in-patient departments for diagnosis $(1,450 / 4,389 ; 33 \%$ versus $668 / 1,939 ; 34.5 \%)$ with no statistically significant differences between 2011 and $2012(\mathrm{p}=0.44)$, while the proportion of TB cases detected among all TB suspects referred to in-patient departments for diagnosis slightly decreased $(551 / 1,450 ; 38 \%$ versus $234 / 668 ; 35 \% ; \mathrm{p}=0.4)$.

The proportion of TB patients diagnosed in TB out-patient units out of the total number registered significantly decreased from $45.2 \%$ to $35.6 \%$ $(527 / 1,165$ versus $273 / 766 ; p<0.001)$. Furthermore, $63 \%$ of TB suspects referred for diagnosis to hospitals $(1,333 / 2,118)$ in the study period were diagnosed as negative for $\mathrm{TB}$.

During the study period, the proportion of patients referred to hospitals to initiate their treatment was $97 \%$ for sputum smear-positive $(n=607)$ and $90 \%$ for sputum smear-negative and extra-pulmonary ( $\mathrm{n}=$ $1,755)$ patients. The proportion of sputum smearpositive cases represented $25.7 \%(607 / 2,362)$ of the total number of registered cases; therefore, most of the hospitalized patients were sputum smear-negative and extra-pulmonary cases.

The costs for a single day of hospital admission in Armenia (2012 costs; 2011 notification data) and the potential savings that can be achieved for each day of inpatient management avoided (in favour of outpatient management) are summarised in the Figure. With a feasible reduction of hospital admission of $75 \%$ of these cases, about 20,000 € might be saved every day, for a total of $7,260,945 €$ per year.

\section{Discussion}

The potential savings that Armenia can re-invest in the TB programme are significant. Established clinical traditions which are prevalent in FSU countries, such as hospitalising non-infectious TB cases for the intensive phase of treatment ( 2 months) and admitting TB suspects for further investigations, do not comply with international recommendations, and pose infection control problems on top of generating unnecessary costs $[1,2,3,4]$. In addition, unnecessary 
Figure. Potential cost reductions and savings (in $€$ ) for each day of hospitalisation avoided in non-infectious TB cases (sputum smear negative and extrapulmonary) using Armenia 2011 notification rates as baseline

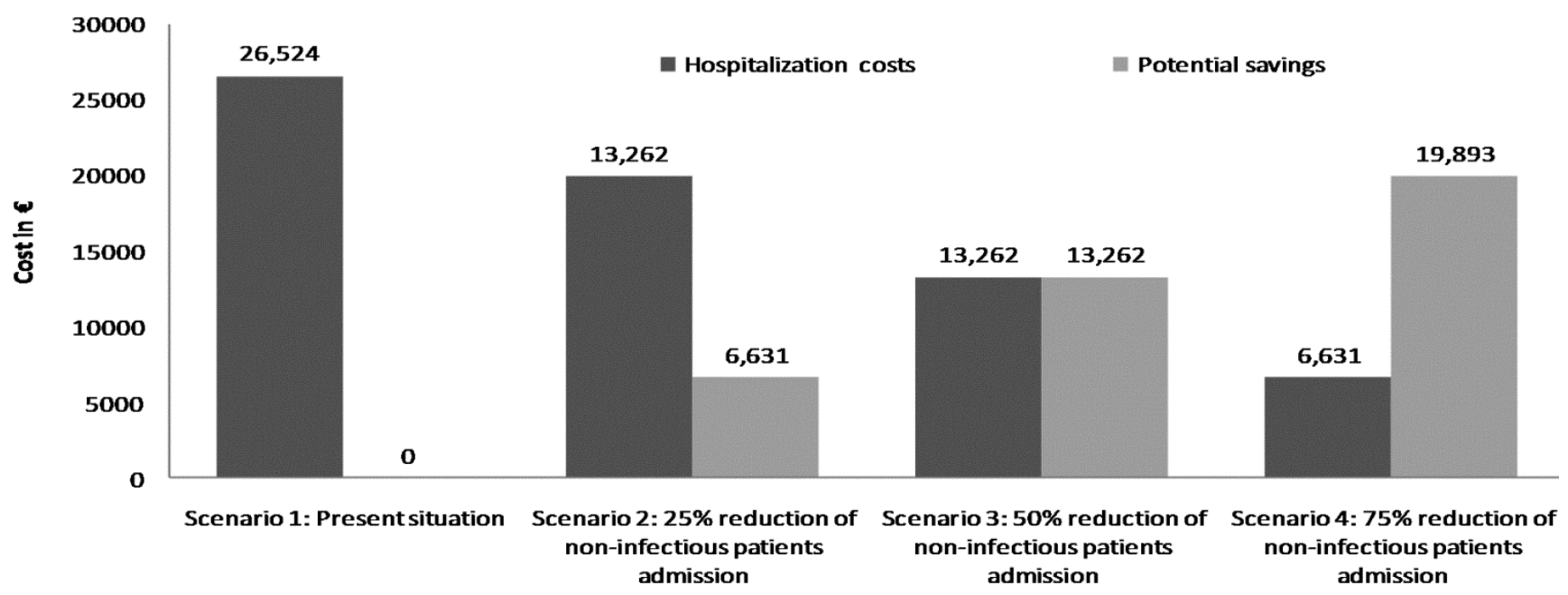

Scenario 1: present situation; Scenario 2: $25 \%$ of non-infectious TB cases not admitted; Scenario 3: $50 \%$ of non-infectious TB cases not admitted; Scenario 4: 75\% of non-infectious TB cases not admitted

hospital admission imposes financial and psychosocial burdens for TB patients.

In Armenia over one third of TB suspects are referred to TB hospitals (by law, for seven days) for diagnosis, with only $35 \%$ of them being diagnosed with active TB.

The study results demonstrate, for the first time in a FSU setting, the savings which can be achieved by decreasing the load of patients sent to hospitals for diagnostic purposes and increasing the proportion of TB patients diagnosed in TB out-patient units.

Furthermore, the Armenian experience shows that no policy change in this direction can be achieved if health-care TB funding mechanisms are not modified. The per-bed daily refund scheme blocks any possible reform of TB admission policies [1].

The economic savings that such a change will generate could be directed to strengthen human resources at the primary health-care level, with improved quality of life for patients and reduced risk of nosocomial transmission $[1,2,3,4]$.

\section{References}

1. Migliori GB, Khomenko AG, Punga VV, Ambrosetti M, Danilova I, Ribka LN, Grzemska M, Sawert H, Raviglione MC (1998) Cost-effectiveness analysis of tuberculosis control policies in Ivanovo Oblast, Russian Federation. Ivanovo Tuberculosis Project Study Group. Bull World Health Organ. 76: 475-483.
2. Falzon D, Jaramillo E, Schünemann HJ, Arentz M, Bauer M, Bayona J, Blanc L, Caminero JA, Daley CL, Duncombe C, Fitzpatrick C, Gebhard A, Getahun H, Henkens M, Holtz TH, Keravec J, Keshavjee S, Khan AJ, Kulier R, Leimane V, Lienhardt C, Lu C, Mariandyshev A, Migliori GB, Mirzayev F, Mitnick CD, Nunn P, Nwagboniwe G, Oxlade O, Palmero D, Pavlinac P, Quelapio MI, Raviglione MC, Rich ML, Royce S, Rüsch-Gerdes S, Salakaia A, Sarin R, Sculier D, Varaine F, Vitoria M, Walson JL, Wares F, Weyer K, White RA, Zignol M (2011) WHO guidelines for the programmatic management of drug-resistant tuberculosis: 2011 update. Eur Respir J 38: 516-528.

3. Migliori GB, Zellweger JP, Abubakar I, Ibraim E, Caminero JA, De Vries G, D'Ambrosio L, Centis R, Sotgiu G, Menegale O, Kliiman K, Aksamit T, Cirillo DM, Danilovits M, Dara M, Dheda K, Dinh-Xuan AT, Kluge H, Lange C, Leimane V, Loddenkemper R, Nicod LP, Raviglione MC, Spanevello A, Thomsen VØ, Villar M, Wanlin M, Wedzicha JA, Zumla A, Blasi F, Huitric E, Sandgren A, Manissero D (2012) European union standards for tuberculosis care. Eur Respir J 39: 807819.

4. Migliori GB, Sotgiu G, D'Ambrosio L, Centis R, Lange C, Bothamley G, Cirillo DM, De Lorenzo S, Guenther G, Kliiman K, Muetterlein R, Spinu V, Villar M, Zellweger JP, Sandgren A, Huitric E, Manissero D (2012) TB and MDR/XDR-TB in European Union and European Economic Area countries: managed or mismanaged? Eur Respir J 39: 619-625.

5. National Statistical Service of the Republic of Armenia. Statistical Yearbook of Armenia 2011 (2011) Yerevan: National Statistical Service of the Republic of Armenia. 624 p.

6. World Health Organization. Global tuberculosis report 2012 (2012) Document WHO/HTM/TB/2012.6. Geneva, World Health Organization. 
7. World Health Organization. Regional Office for Europe. Dara M, Mkrtchyan Z, Ghukasyan G (2011) Extensive review of TB prevention, care and control services Tuberculosis programme in Armenia, 21 April - 4 May 2011. Copenhagen: WHO Regional Office for Europe. $80 \mathrm{p}$.

8. World Health Organization. Regional Office for Europe (2011) Roadmap to prevent and combat drug-resistant tuberculosis: the consolidated action plan to prevent and combat multidrug- and extensively drug-resistant tuberculosis in the WHO European Region 2011-2015. Copenhagen: WHO Regional Office for Europe. 103 p.

9. Truzyan N, Grigoryan R, Martirosyan H, Petrosyan V, Crape B, Melkom Melkomian D, Koshkakaryan M, Tadevosyan M, Harutyunyan T (2012) Operational Research on Investigation of TB Risk Factors in Armenia. Yerevan: Center for Health Services Research and Development, American University of Armenia; vi, $81 \mathrm{p}$.

10. Ministry of Health of Armenia (2012) Free in-patient and outpatient Medical, Hygienic and Untiepidemic service costs and Normatives of Republic of Armenia 2012. Yerevan: Ministry of Health of Armenia.

\section{Corresponding author}

Giovanni Sotgiu

Epidemiology and Medical Statistics Unit

Department of Biomedical Sciences

University of Sassari

Research, Medical Education and Professional Development Unit AOU Sassari, Italy

Via Padre Manzella, 4

Sassari, 07100, Italy

Telephone: +39 079 229959; Fax: +39 079228472

Email: gsotgiu@uniss.it

Conflict of interests: No conflict of interests is declared. 